\title{
VISION LOSS FOLLOWING SNAKEBITE IN A PATIENT WITH CONTROLLED APLASTIC ANEMIA
}

\section{Kweon EY (1), Lee DW (1), Ahn M (1), Nork TM (2), Cho NC (1)}

(1) Department of Ophthalmology, Chonbuk National University Medical School, Jeonju, Jeonbuk, South Korea; (2) Department of Ophthalmology and Visual Sciences, University of Wisconsin Medical School, Madison, Wisconsin, USA.

ABSTRACT: Viper venoms act mainly on blood and blood vessels. Reports of ophthalmic manifestations after snakebite include ptosis and ophthalmoplegia. In the current study, we describe a case that developed bilateral retinal and subretinal hemorrhage following snakebite. Bilateral retinal hemorrhage is a rare ocular complication of snake envenomation and has not been reported with fundus photographs in the literature so far.

KEY WORDS: snakebite, retinal hemorrhage, visual loss, aplastic anemia.

CONFLICTS OF INTEREST: There is no conflict.

\section{CORRESPONDENCE TO:}

NAM CHUN CHO, Department of Ophthalmology, Chonbuk National University Medical School, San 2-20 Geumam-dong, Deokjin-gu, Jeonju, Jeonbuk, 561-180, South Korea. Phone: 8263250 1969. Fax: 8263250 1960. Email: cnauo@chonbuk.ac.kr. 


\section{INTRODUCTION}

Venomous snakebites may result in hemostatic or neurological dysfunction. Ocular complications are very rare, but common ocular involvements are generally connected with neurological ones. Visual loss subsequent to snakebite may result from neurological or hemostatic abnormalities as well as from direct ocular inoculations (1).

We report a case of a patient with controlled aplastic anemia which developed visual loss after bilateral retinal and subretinal hemorrhages following snakebite. Bilateral retinal hemorrhage is an infrequent ocular effect caused by snakebite and has not been previously reported in the literature.

\section{CASE REPORT}

A 62-year-old Korean woman presented blurred vision in both eyes. The patient had been diagnosed with aplastic anemia one year before and maintained relatively stable blood cell and platelet counts without requiring transfusion. Just one month previous to the snakebite, her blood counts were: hemoglobin $5.8 \mathrm{~g} / \mathrm{dL}$, hematocrit $17.7 \%$ and 10,000 platelets/ $\mu \mathrm{L}$. While working in a forest she was bitten by a snake on her right leg and one week after this accident, she lost her vision. The snake was brownish, presumably a viper; since these animals are common in the region. She was treated with anti-snake venom (ASV) at a local clinic.

The right leg showed severe swelling and subcutaneous hemorrhage (Figure 1). Her best corrected visual acuity (BCVA) was 20/400 in both eyes. The intraocular pressures were $19 \mathrm{mmHg}$ (right eye) and $18 \mathrm{mmHg}$ (left eye). Anterior segment examination of both eyes was unremarkable. Fundus examination of the eyes showed profound retinal and subretinal hemorrhages with Roth's spots (Figure 2). Fluorescein angiography showed blocked fluorescence due to retinal and subretinal hemorrhage (Figure 2). Blood tests revealed hemoglobin value of $4.9 \mathrm{~g} / \mathrm{dL}$, hematocrit of $15.2 \%, 2,000$ platelets $/ \mu \mathrm{L}$ and increased titers of fibrin degradation products (FDP) and D-dimer. After transfusion, her blood counts returned to normal values: hemoglobin $8.1 \mathrm{~g} / \mathrm{dL}$, hematocrit $23.3 \%$ and 21,000 platelets/ $\mu \mathrm{L}$.

After two months her BCVA had improved to 20/100 in the right eye and 20/60 in the left one. However, funduscopic examination showed persistent retinal and subretinal hemorrhage in both eyes (Figure 3). 

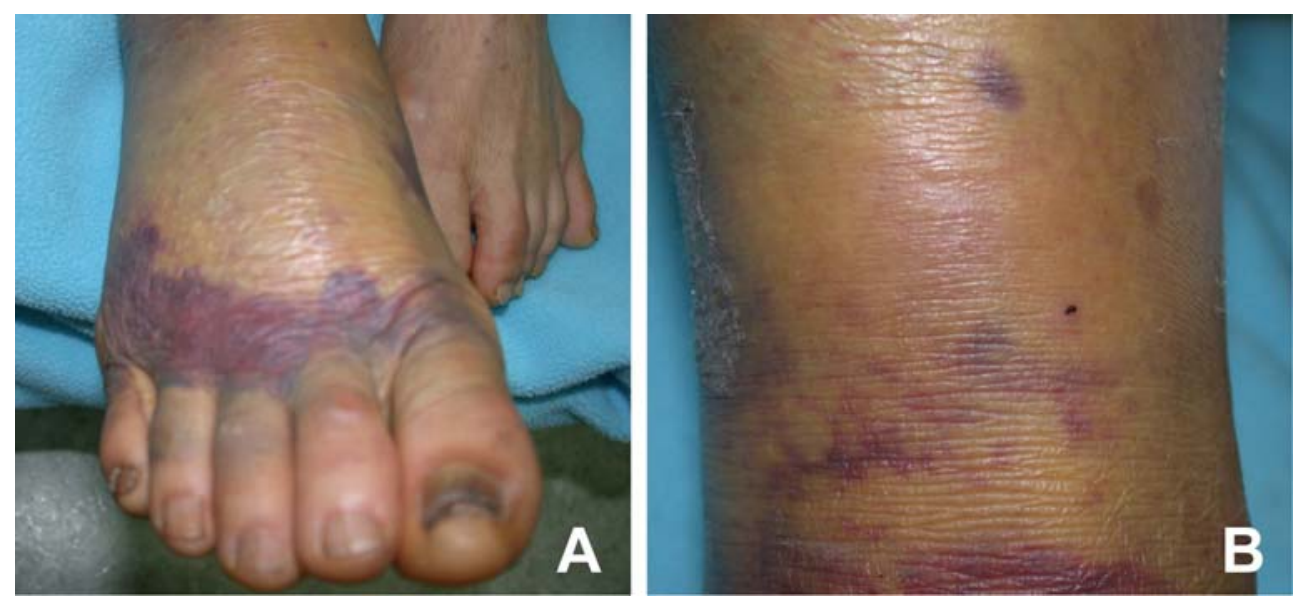

Figure 1. Right leg showing swelling and subcutaneous hemorrhage.
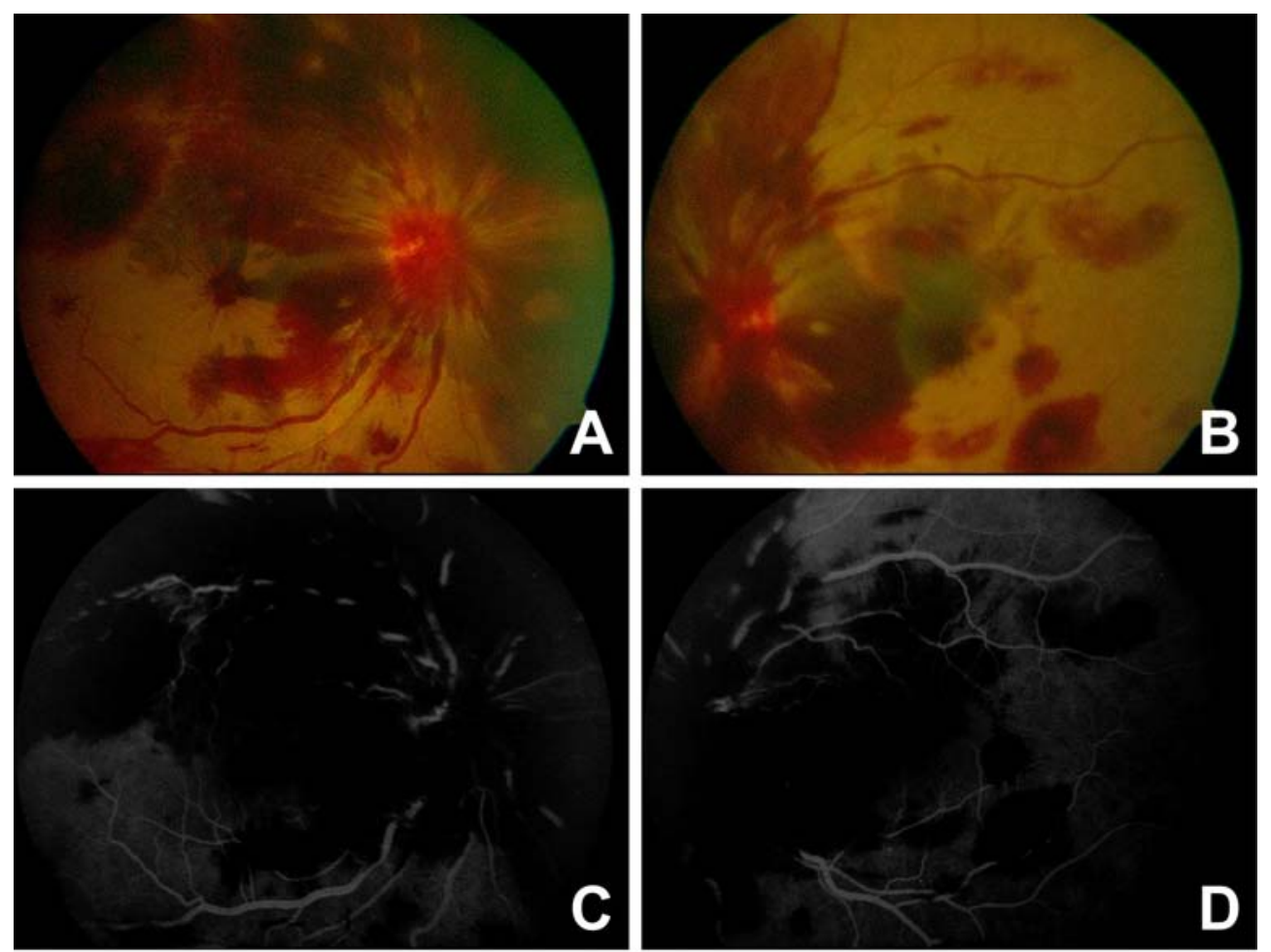

Figure 2. Right (A) and left (B) fundus at initial examination. Fluorescein angiography of right (C) and left (D) fundus at initial examination. Note profound retinal and subretinal hemorrhages with Roth's spots. Flourescein angiography showed blocked fluorescence. 


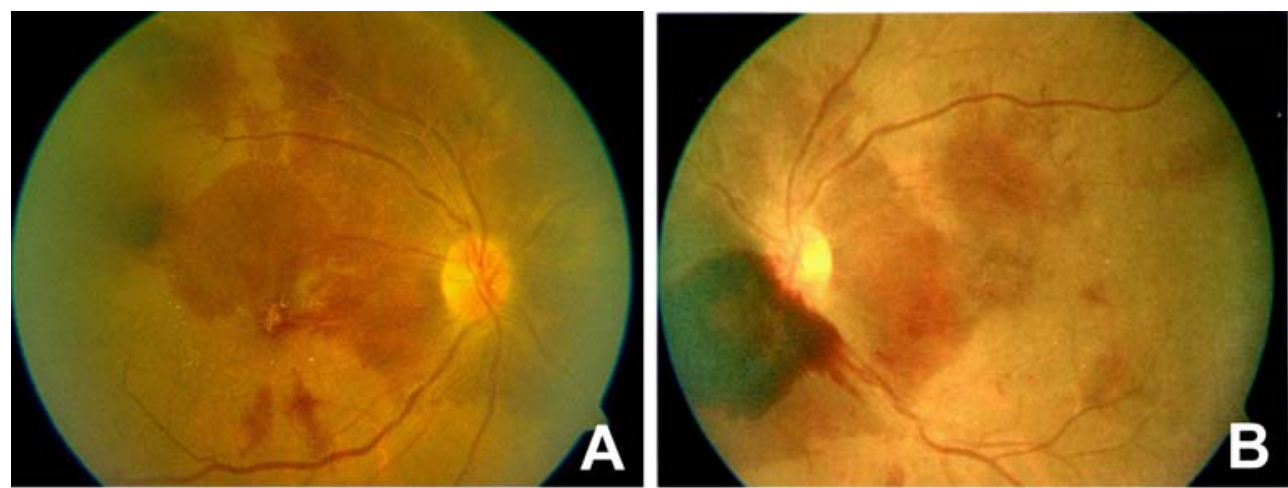

Figure 3. Right (A) and left (B) fundus two months after initial examination. Note the persistent retinal and subretinal hemorrhages in both eyes.

\section{DISCUSSION}

Snake venom is a complex mixture of proteins which affects both hemostatic and neurologic systems (2). General manifestations of snakebites depend on specific toxins that constitute the venom. Antihemostatic factors of viper venoms can lead to acute fibrinolysis, severe reduction of platelet levels and damage to vascular endothelium. Viper venoms may also cause breakdown of permeability barriers provoking fluid extravasation and edema. Peripheral neutrophil count can increase up to 20,000 cells/ $\mu \mathrm{L}$ or more in severely envenomed patients. Initial hemoconcentration, a consequence of plasma extravasation, is followed by anemia caused by bleeding or, more rarely, hemolysis. Thrombocytopenia is a common effect of pit viper bites. Hyaluronidase and collagenase, proteolytic enzymes that are also compounds of viper venoms, may induce disruption of retinal veins that, in turn, result in retinal hemorrhage.

Although the snake was not identified in our patient, one can presume from the hemotoxic symptoms that the animal could have been a member of Viperidae family. Swelling and bruising of the bitten limb result from increased vascular permeability produced by proteases, phospholipases, membrane-damaging polypeptide toxins and endogenous autacoids released by venom, such as histamine, shydroxytryptamine and kinin. The underlying and presumably controlled aplastic anemia in our patient may have exacerbated the response to the bite.

Ophthalmic manifestations of snakebite have rarely been reported in the literature. However, the common problem described after snakebites is a neurological disturbance in the form of ophthalmoplegia (3). Other ocular disturbances observed as effects of snakebites range from keratomalacia to vitreous hemorrhage, including uveitis, glaucoma, central retinal artery occlusion, macular infarction, optic neuritis, penetrating eye injury, globe necrosis and visual loss due to cortical infarction (1, 37). The signs, symptoms and magnitude of pit viper envenomation depend on 
multiple factors, such as patient age, presence of underlying diseases, and time interval between the bite and treatment.

In this case, the patient with controlled aplastic anemia developed visual loss after bilateral retinal and subretinal hemorrhage following the snakebite. We believe that the cause of this rare complication is the synergistic effect between the envenomation and her systemic disease.

\section{REFERENCES}

1. Bhalla A, Jain AP, Banait S, Jajoo UN, Kalantri SP. Central retinal artery occlusion: an unusual complication of snakebite. J Venom Anim Toxins incl Trop Dis. 2004;10(3):311-4.

2. Marsh NA. Snake venoms affecting the haemostatic mechanism: a consideration of their mechanisms, practical applications and biological significance. Blood Coagul Fibrinolysis. 1994;5(3):399-410.

3. Takeshita T, Yamada K, Hanada M, Oda-Ueda N. Extraocular muscle paresis caused by snakebite. Kobe J Med Sci. 2003;49(1-2):11-5.

4. Menon V, Tandon R, Sharma T, Gupta A. Optic neuritis following snake bite. Indian J Ophthalmol. 1997;45(4):236-7.

5. Singh J, Singh P, Singh R, Vig VK. Macular infarction following viperine snake bite. Arch Ophthalmol. 2007;125(10):1430-1.

6. Hayreh SS. Transient central retinal artery occlusion following viperine snake bite. Arch Ophthalmol. 2008;126(6):870-1

7. Rao BM. A case of bilateral vitreous haemorrhage following snake bite. Indian J Ophthalmol. 1977;25(2):1-2 